MITSUBISHI ELECTRIC RESEARCH LABORATORIES

http://www.merl.com

\title{
Knowledge-Aided Adaptive Coherence Estimator in Stochastic Partially Homogeneous Environments
}

\author{
Wang, P.; Sahinoglu, Z.; Pun, M-O; Li, H.; Himed, B. \\ TR2011-010 January 2011
}

\begin{abstract}
This paper introduces a stochastic partially homogeneous model for adaptive signal detection. In this model, the disturbance covariance matrix of training signals, $\mathrm{R}$, is assumed to be a random matrix with some a priori information, while the disturbance covariance matrix of the test signal, $\mathrm{R} 0$, is assumed to be equal to $\mathrm{R}$, i.e., $\mathrm{R} 0=\mathrm{R}$. On one hand, this model extends the stochastic homogeneous model by introducing an unknown power scaling factor between the test and training signals. On the other hand, it can be considered as a generalization of the standard partially homogeneous model to the stochastic Bayesian framework, which treats the covariance matrix as a random matrix. According to the stochastic partially homogeneous model, a scale-invariant generalized likelihood ratio test (GLRT) for the adaptive signal detection is developed, which is a knowledge-aided version of the well-known adaptive coherence estimator (ACE). The resulting knowledge-aided ACE (KA-ACE) employs a colored loading step utilizing the a priori knowledge and the sample covariance matrix. Various simulation results and comparison with respect to other detectors confirm the scale-invariance and the effectiveness of the KA-ACE.
\end{abstract}

IEEE Signal Processing Letters

This work may not be copied or reproduced in whole or in part for any commercial purpose. Permission to copy in whole or in part without payment of fee is granted for nonprofit educational and research purposes provided that all such whole or partial copies include the following: a notice that such copying is by permission of Mitsubishi Electric Research Laboratories, Inc.; an acknowledgment of the authors and individual contributions to the work; and all applicable portions of the copyright notice. Copying, reproduction, or republishing for any other purpose shall require a license with payment of fee to Mitsubishi Electric Research Laboratories, Inc. All rights reserved. 



\title{
Knowledge-Aided Adaptive Coherence Estimator in Stochastic Partially Homogeneous Environments
}

\author{
$\mathrm{Pu}$ Wang, Zafer Sahinoglu, Man-On Pun, Hongbin Li, and Braham Himed
}

\begin{abstract}
This paper introduces a stochastic partially homogeneous model for adaptive signal detection. In this model, the disturbance covariance matrix of training signals, $R$, is assumed to be a random matrix with some $a$ priori information, while the disturbance covariance matrix of the test signal, $\mathbf{R}_{0}$, is assumed to be equal to $\lambda \mathbf{R}$, i.e., $\mathbf{R}_{0}=\lambda \mathbf{R}$. On one hand, this model extends the stochastic homogeneous model by introducing an unknown power scaling factor $\lambda$ between the test and training signals. On the other hand, it can be considered as a generalization of the standard partially homogeneous model to the stochastic Bayesian framework, which treats the covariance matrix as a random matrix. According to the stochastic partially homogeneous model, a scale-invariant generalized likelihood ratio test (GLRT) for the adaptive signal detection is developed, which is a knowledge-aided version of the well-known adaptive coherence estimator (ACE). The resulting knowledge-aided ACE (KA-ACE) employs a colored loading step utilizing the $a$ priori knowledge and the sample covariance matrix. Various simulation results and comparison with respect to other detectors confirm the scale-invariance and the effectiveness of the KA-ACE.
\end{abstract}

Index Terms - Partially homogeneous model, knowledge-aided, generalized likelihood ratio test, Bayesian inference.

\section{INTRODUCTION}

For the adaptive signal detection problem, a homogeneous environment is usually assumed, where the test signal shares the same covariance matrix with the training signals [1], [2]. Recently, a Bayesian approach to the detection problem emerged [3], [4], where the covariance matrix is assumed to be randomly distributed with some prior distribution. The resulting detectors are often referred to as knowledgeaided (KA) detectors for the stochastic homogeneous environment. Using both measured L-band clutter data and highfidelity KASSPER data, the KA detectors were shown to have improved performance than the conventional detectors when the homogeneous training signals are limited [4]. For non-homogeneous environments, several models have been proposed. One of these models is the compound-Gaussian model, in which a power-varying texture component across range bins is used to characterize the heavy-tailed clutter distributions in radar, especially for sea clutter. Another model is a partially homogeneous model, where the training signals share the covariance matrix with the test signal up to an

P. Wang and H. Li are with the Department of Electrical and Computer Engineering, Stevens Institute of Technology, Hoboken, NJ 07030, USA (email: \{pwang4, Hongbin.Li\}@stevens.edu).

Z. Sahinoglu and M. O. Pun are with Mitsubishi Electric Research Laboratories (MERL), 201 Broadway, Cambridge, MA 02139, USA (e-mail: \{zafer, mpun\}@merl.com).

B. Himed is with AFRL/RYRT, Dayton, OH 45433 (e-mail: Braham.Himed@wpafb.af.mil). unknown scaling factor [5]-[8]. A recent addition to the nonhomogeneous model is a stochastic heterogeneous model [9][11], in which two layers of random matrices are used to model the heterogeneity between the test and training signals. This model includes not only the power variation across the range, but also the structural difference of the covariance matrix.

In this paper, we consider the partially homogeneous model, which has received much attention over the last decade [5]-[8]. One motivation to consider the partially homogeneous model is due to the use of guard cells in radar signal processing. In array signal processing and space-time adaptive processing (STAP), a number of guard cells are often used to mitigate the sidelobe effects and hence separate the test signal and training signals, which may lead to a power difference between the test and training signals [12]. A stochastic partially homogeneous model is proposed in this paper, which is different than the standard partially homogeneous model. This model allows us to incorporate some a priori knowledge of the environment, while also retaining the heterogeneity between the test and training signals by using the power scaling factor. Specifically, we consider the following hypothesis testing problem [5]-[8]:

$$
\begin{aligned}
& H_{0}: \quad \mathbf{x}_{0}=\mathbf{d}_{0}, \quad \mathbf{x}_{k}=\mathbf{d}_{k}, k=1, \cdots, K, \\
& H_{1}: \quad \mathbf{x}_{0}=\alpha \mathbf{s}+\mathbf{d}_{0}, \quad \mathbf{x}_{k}=\mathbf{d}_{k}, k=1, \cdots, K,
\end{aligned}
$$

where $\mathbf{x}_{0} \in \mathbb{C}^{N \times 1}$ is the test signal, $\mathbf{x}_{k}=\mathbf{d}_{k}, k=1, \cdots, K$, are target-free training signals, $\mathbf{s}$ is the known array response, $\alpha$ is an unknown complex-valued amplitude, $\mathbf{d}_{0}$ and $\mathbf{d}_{k}$ are independent, zero-mean complex-valued Gaussian distributed random vectors with covariance matrices given by

$$
E\left\{\mathbf{d}_{0} \mathbf{d}_{0}^{H}\right\}=\mathbf{R}_{0}=\lambda \mathbf{R}, \quad E\left\{\mathbf{d}_{k} \mathbf{d}_{k}^{H}\right\}=\mathbf{R},
$$

where $\lambda$ is an unknown power scaling factor. Furthermore, we assume the covariance matrix $\mathbf{R}$ to be random and has a complex inverse Wishart distribution, i.e., $\mathbf{R} \sim \mathcal{C W}^{-1}((\mu-$ $N) \overline{\mathbf{R}}, \mu)$ [3], [4], [9]:

$$
p(\mathbf{R})=\frac{|(\mu-N) \overline{\mathbf{R}}|^{\mu}}{\tilde{\Gamma}(N, \mu)|\mathbf{R}|^{\mu+N}} e^{-(\mu-N) \operatorname{tr}\left(\mathbf{R}^{-1} \overline{\mathbf{R}}\right)},
$$

where $\tilde{\Gamma}(N, \mu)=\pi^{N(N-1) / 2} \prod_{k=1}^{N} \Gamma(\mu-N+k)$ with $\Gamma(\cdot)$ denoting the Gamma function and $\overline{\mathbf{R}}$ the known prior covariance matrix which can be obtained from sources such as land-cover/land-use (LCLU) maps, past measurements, etc. [11]. The parameter $\mu$ indicates the importance of the prior knowledge $\overline{\mathbf{R}}$. The larger $\mu$ is, the more important $\overline{\mathbf{R}}$ is. Since $\mathbf{R}_{0}=\lambda \mathbf{R}$, it is straightforward to show that $\mathbf{R}_{0} \sim$ $\mathcal{C W}^{-1}((\mu-N) \lambda \overline{\mathbf{R}}, \mu)$. If $\lambda=1$, the stochastic partially 
homogeneous model reduces to the stochastic homogeneous model [3], [4].

According to the stochastic partially homogeneous model, the scale-invariant generalized likelihood ratio test (GLRT) is developed within a Bayesian framework. The likelihood function is first obtained by averaging the conditional likelihood function w.r.t. the prior distribution of the covariance matrix. Then, maximization of the likelihood function is performed with respect to the deterministic parameters, namely the scaling factor $\lambda$ and the amplitude $\alpha$. Finally, the generalized likelihood ratio test is derived in closed-form. The resulting scale-invariant GLRT is a knowledge-aided (KA) version of the adaptive coherence estimator (ACE) of [5], which is referred to as the KA-ACE. Specifically, the proposed KA-ACE uses a linear combination of the sample covariance matrix and the a priori matrix $\overline{\mathbf{R}}$, where the amount of loading $\overline{\mathbf{R}}$ is controlled by the parameter $\mu$, which reflects the accuracy of the prior $\overline{\mathbf{R}}$. Moreover, the scale-invariance property of the $\mathrm{KA}-\mathrm{ACE}$ and numerical comparisons with other detectors are investigated in this paper.

\section{Generalized LiKelihood RATIO TEST FOR Stochastic Partially HoMOGENEOUS ENVIRONMENT}

In the following, the likelihood function which involves maximization with respect to $\lambda$ and maximization with respect to $\alpha$ is discussed.

\section{A. Likelihood Ratio Test}

The KA-ACE is developed from a Bayesian framework which takes the form as

$$
T=\frac{\max _{\alpha, \lambda} \int f_{1}\left(\mathbf{x}_{0}, \mathbf{x}_{1}, \cdots, \mathbf{x}_{K} \mid \alpha, \lambda, \mathbf{R}\right) p(\mathbf{R}) d \mathbf{R}}{\max _{\lambda} \int f_{0}\left(\mathbf{x}_{0}, \mathbf{x}_{1}, \cdots, \mathbf{x}_{K} \mid \lambda, \mathbf{R}\right) p(\mathbf{R}) d \mathbf{R}}
$$

where

$$
\begin{aligned}
& f_{i}\left(\mathbf{x}_{0}, \mathbf{x}_{1}, \cdots, \mathbf{x}_{K} \mid \alpha, \lambda, \mathbf{R}\right), \quad i=0,1 \\
= & f_{i}\left(\mathbf{x}_{0} \mid \alpha, \lambda, \mathbf{R}\right) f\left(\mathbf{x}_{1}, \cdots, \mathbf{x}_{K} \mid \mathbf{R}\right) \\
= & \frac{1}{\pi^{(K+1) N} \lambda^{N}|\mathbf{R}|^{K+1}} \exp \left\{-\operatorname{tr}\left(\mathbf{R}^{-1} \boldsymbol{\Sigma}_{i}\right)\right\},
\end{aligned}
$$

and

$$
\boldsymbol{\Sigma}_{i}=\lambda^{-1} \mathbf{y}_{i} \mathbf{y}_{i}^{H}+\mathbf{S}
$$

with $\mathbf{y}_{i}=\mathbf{x}_{0}-\beta_{i} \alpha \mathbf{s}, \beta_{1}=1, \beta_{0}=0$, and $\mathbf{S}=\sum_{k=1}^{K} \mathbf{x}_{k} \mathbf{x}_{k}^{H}$.

The likelihood function can be obtained by averaging the conditional likelihood function w.r.t. the prior distribution as

$$
\begin{aligned}
& \int f_{i}\left(\mathbf{x}_{0}, \mathbf{x}_{1}, \cdots, \mathbf{x}_{K} \mid \alpha, \lambda, \mathbf{R}\right) p(\mathbf{R}) d \mathbf{R} \\
= & \frac{|(\mu-N) \overline{\mathbf{R}}|^{\mu}}{\pi^{(K+1) N} \lambda^{N} \tilde{\Gamma}(N, \mu)} \int|\mathbf{R}|^{-(L+N)} e^{-\operatorname{tr}\left(\mathbf{R}^{-1} \overline{\mathbf{\Sigma}}_{i}\right)} d \mathbf{R} \\
= & \frac{|(\mu-N) \overline{\mathbf{R}}|^{\mu} \tilde{\Gamma}(N, K+\mu+1)}{\pi^{(K+1) N} \tilde{\Gamma}(N, \mu) \lambda^{N}}\left|\overline{\mathbf{\Sigma}}_{i}\right|^{-L},
\end{aligned}
$$

where $L=K+\mu+1$, and

$$
\overline{\boldsymbol{\Sigma}}_{i}=\boldsymbol{\Sigma}_{i}+(\mu-N) \overline{\mathbf{R}}=\lambda^{-1} \mathbf{y}_{i} \mathbf{y}_{i}^{H}+\mathbf{S}+(\mu-N) \overline{\mathbf{R}} .
$$

The likelihood function incorporates the prior knowledge $\overline{\mathbf{R}}$ and retains the information from the sample covariance matrix S. With (7), the likelihood ratio test of (4) reduces to

$$
T=\frac{\max _{\alpha} \max _{\lambda} \lambda^{-N}\left|\overline{\boldsymbol{\Sigma}}_{1}\right|^{-L}}{\max _{\lambda} \lambda^{-N}\left|\overline{\boldsymbol{\Sigma}}_{0}\right|^{-L}} .
$$

\section{B. Maximization over the Scaling Factor $\lambda$}

From (9), the maximum likelihood (ML) estimate of the scaling factor $\lambda$ is

$$
\begin{aligned}
\hat{\lambda}_{i} & =\arg \max _{\lambda} \lambda^{-N}\left|\overline{\boldsymbol{\Sigma}}_{i}\right|^{-L}, \quad i=0,1, \\
& =\arg \min _{\lambda} \lambda^{N}\left|\lambda^{-1} \mathbf{y}_{i} \mathbf{y}_{i}^{H}+\mathbf{S}+(\mu-N) \overline{\mathbf{R}}\right|^{L} .
\end{aligned}
$$

Let $\boldsymbol{\Xi}=\mathbf{S}+(\mu-N) \overline{\mathbf{R}}$. The above cost function can be simplified as

$$
\begin{aligned}
& \lambda^{N}\left|\lambda^{-1} \mathbf{y}_{i} \mathbf{y}_{i}^{H}+\boldsymbol{\Xi}\right|^{L}=\lambda^{N}|\boldsymbol{\Xi}|^{L}\left|\lambda^{-1} \boldsymbol{\Xi}^{-1} \mathbf{y}_{i} \mathbf{y}_{i}^{H}+\mathbf{I}\right|^{L} \\
& =|\boldsymbol{\Xi}|^{L} \lambda^{N}\left(1+\lambda^{-1} \mathbf{y}_{i}^{H} \boldsymbol{\Xi}^{-1} \mathbf{y}_{i}\right)^{L}
\end{aligned}
$$

Taking the log-derivative and setting it to zero, we have

$$
N-L \frac{\mathbf{y}_{i}^{H} \boldsymbol{\Xi}^{-1} \mathbf{y}_{i}}{\lambda+\mathbf{y}_{i}^{H} \boldsymbol{\Xi}^{-1} \mathbf{y}_{i}}=0,
$$

which gives the ML estimate of $\lambda$

$$
\hat{\lambda}_{\mathrm{ML}, i}=\frac{L-N}{N} \mathbf{y}_{i}^{H} \boldsymbol{\Xi}^{-1} \mathbf{y}_{i} .
$$

The cost function reduces to

$$
\min _{\lambda} \lambda^{N}\left|\overline{\boldsymbol{\Sigma}}_{i}\right|^{L}=\left(\frac{L}{L-N}\right)^{L}|\boldsymbol{\Xi}|^{L} \hat{\lambda}_{\mathrm{ML}, i}^{N}
$$

Therefore, the generalized likelihood function becomes

$$
\begin{aligned}
T & =\max _{\alpha} \frac{\hat{\lambda}_{\mathrm{ML}, 0}^{N}\left|\overline{\mathbf{\Sigma}}_{0}\left(\hat{\lambda}_{\mathrm{ML}, 0}\right)\right|^{L}}{\hat{\lambda}_{\mathrm{ML}, 1}^{N}\left|\overline{\mathbf{\Sigma}}_{1}\left(\alpha, \hat{\lambda}_{\mathrm{ML}, 1}\right)\right|^{L}} \\
& =\left[\frac{\mathbf{x}_{0}^{H} \boldsymbol{\Xi}^{-1} \mathbf{x}_{0}}{\min _{\alpha}\left(\mathbf{x}_{0}-\alpha \mathbf{s}\right)^{H} \mathbf{\Xi}^{-1}\left(\mathbf{x}_{0}-\alpha \mathbf{s}\right)}\right]^{N} .
\end{aligned}
$$

\section{Maximization over the Amplitude $\alpha$}

By minimizing the term $\left(\mathbf{x}_{0}-\alpha \mathbf{s}\right)^{H} \boldsymbol{\Xi}^{-1}\left(\mathbf{x}_{0}-\alpha \mathbf{s}\right)$, the ML estimate of $\alpha$ is given by [1, the fourth equation on p. 118]

$$
\hat{\alpha}_{\mathrm{ML}}=\frac{\mathbf{s}^{H} \boldsymbol{\Xi}^{-1} \mathbf{x}_{0}}{\mathbf{s}^{H} \boldsymbol{\Xi}^{-1} \mathbf{s}},
$$

and the minimum cost function is

$$
\min _{\alpha}\left(\mathbf{x}_{0}-\alpha \mathbf{s}\right)^{H} \boldsymbol{\Xi}^{-1}\left(\mathbf{x}_{0}-\alpha \mathbf{s}\right)=\mathbf{x}_{0}^{H} \boldsymbol{\Xi}^{-1} \mathbf{x}_{0}-\frac{\left|\mathbf{s}^{H} \boldsymbol{\Xi}^{-1} \mathbf{x}_{0}\right|^{2}}{\mathbf{s}^{H} \boldsymbol{\Xi}^{-1} \mathbf{s}} \text {. }
$$

Taking the $N$-th square root of (15) and utilizing the monotonic property of the function $f(x)=1 /(1-x)$, we obtain the KA-ACE statistic as

$$
T_{\mathrm{KA}-\mathrm{ACE}}=\frac{\left|\mathbf{s}^{H} \boldsymbol{\Xi}^{-1} \mathbf{x}_{0}\right|^{2}}{\left(\mathbf{s}^{H} \boldsymbol{\Xi}^{-1} \mathbf{s}\right)\left(\mathbf{x}_{0}^{H} \boldsymbol{\Xi}^{-1} \mathbf{x}_{0}\right)} \underset{H_{0}}{\stackrel{H_{1}}{\gtrless}} \gamma_{\mathrm{KA} \text {-ACE }}
$$


where $\gamma_{\mathrm{KA}-\mathrm{ACE}}$ denotes a threshold set by a chosen probability of false alarm. It is seen that the KA-ACE for the stochastic partially homogeneous environment takes the same form as that of the standard ACE [5], except that the whitening matrix is given by

$$
\boldsymbol{\Xi}=\mathbf{S}+(\mu-N) \overline{\mathbf{R}}=\sum_{k=1}^{K} \mathbf{x}_{k} \mathbf{x}_{k}^{H}+(\mu-N) \overline{\mathbf{R}} .
$$

which uses a linear combination of the sample covariance matrix $\mathbf{S}$ and the prior knowledge $\overline{\mathbf{R}}$. The weighting factor of $\overline{\mathbf{R}}$ is controlled by $\mu$. Specifically, the KA-ACE puts more weight on $\overline{\mathbf{R}}$, when the prior matrix is more accurate (i.e., $\mu$ is large). In comparison, the standard ACE takes the form of (18) but with the whitening matrix given by the sample covariance matrix $\boldsymbol{\Xi}=\mathbf{S}$. It is interesting to note that the KA-ACE can also be derived from other heuristic ways:

- The MAP-ACE which exploits the maximum a posteriori (MAP) estimate of $\mathbf{R}$ takes the form

$$
\begin{aligned}
& T_{\text {MAP-ACE }} \\
& =\frac{\max _{\alpha, \lambda, \mathbf{R}}\left\{f_{1}\left(\mathbf{x}_{0}, \mathbf{x}_{1}, \cdots, \mathbf{x}_{K} \mid \alpha, \lambda, \mathbf{R}\right) p(\mathbf{R})\right\}}{\max _{\lambda, \mathbf{R}}\left\{f_{0}\left(\mathbf{x}_{0}, \mathbf{x}_{1}, \cdots, \mathbf{x}_{K} \mid \lambda, \mathbf{R}\right) p(\mathbf{R})\right\}} .
\end{aligned}
$$

It can be shown that the MAP estimate of $\mathbf{R}$ is [4]

$$
\hat{\mathbf{R}}_{\mathrm{MAP}, i}=\frac{\overline{\mathbf{\Sigma}}_{i}}{L+N}, \quad i=0,1
$$

Substituting the MAP estimate into (20), the MAP-ACE takes the same form of (9) and, hence, coincides with the KA-ACE (18) afterwards.

- The MMSE-ACE takes the form of (20) with the minimum mean square error (MMSE) estimate of $\mathbf{R}$ replacing the MAP estimate,

$$
\begin{aligned}
& T_{\text {MMSE - ACE }} \\
& =\frac{\left.\max _{\alpha, \lambda}\left\{f_{1}\left(\mathbf{x}_{0}, \mathbf{x}_{1}, \cdots, \mathbf{x}_{K} \mid \alpha, \lambda, \mathbf{R}\right) p(\mathbf{R})\right\}\right|_{\mathbf{R}=\hat{\mathbf{R}}_{\mathrm{MMSE}, 1}}}{\left.\max _{\lambda}\left\{f_{0}\left(\mathbf{x}_{0}, \mathbf{x}_{1}, \cdots, \mathbf{x}_{K} \mid \lambda, \mathbf{R}\right) p(\mathbf{R})\right\}\right|_{\mathbf{R}=\hat{\mathbf{R}}_{\mathrm{MMSE}, 0}}},
\end{aligned}
$$

where the MMSE estimate of $\mathbf{R}$ is obtained as the mean of the posterior probability [4]

$$
\begin{aligned}
\hat{\mathbf{R}}_{\mathrm{MMSE}, i} & =\int \mathbf{R} f_{i}\left(\mathbf{R} \mid \mathbf{x}_{0}, \mathbf{x}_{1}, \cdots, \mathbf{x}_{K}, \alpha, \lambda\right) d \mathbf{R} \\
& =\frac{\overline{\boldsymbol{\Sigma}}_{i}}{L-N}, \quad i=0,1,
\end{aligned}
$$

which is proportional to the MAP estimate of (20). Therefore, the MMSE-ACE results in the form of (9) and gives the same detection statistic as that of the KA-ACE in (18).

\section{Performance Evaluation}

In this section, simulation results are provided to demonstrate the performance of the KA-ACE detector, which is also compared to other detectors. In all simulation examples, we consider the case where $N=16$ channels and the steering

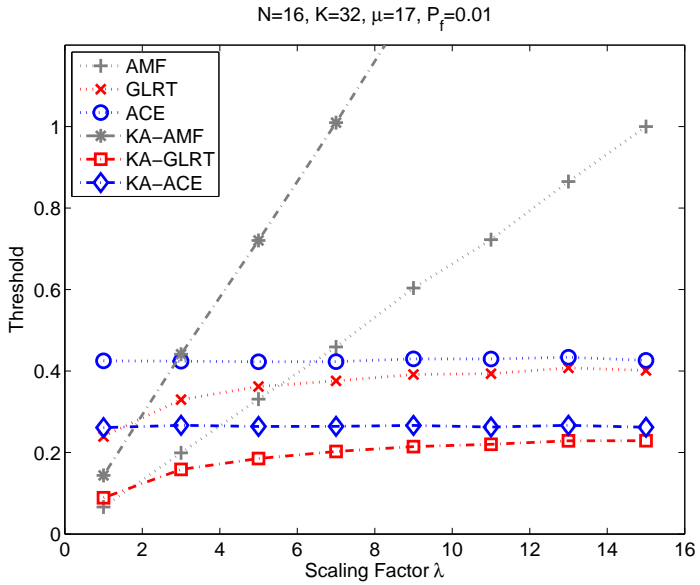

Fig. 1. Scale-Invariance: Threshold versus the scaling factor $\lambda$ for various detectors when $N=16, K=32, \mu=17, P_{f}=0.01$, and SNR $=25 \mathrm{~dB}$.

vector is given by $\mathbf{s}=[1, \cdots, 1]^{T}$. The average signal-tonoise ratio (SNR) is defined as

$$
\mathrm{SNR}=|\alpha|^{2} \mathbf{s}^{H} \overline{\mathbf{R}}^{-1} \mathbf{s},
$$

where $\overline{\mathbf{R}}$ is the mean of the random covariance matrix $\mathbf{R}$. We set the prior covariance matrix $\overline{\mathbf{R}}$ as [9]

$$
[\overline{\mathbf{R}}]_{i j}=\rho^{|i-j|},
$$

where $\rho=0.9$ is chosen. The simulated performance is obtained using 10000 Monte Carlo trials and the probability of false alarm is set to $P_{f}=0.01$. It is noted that these simulation parameters, e.g., the covariance matrix of (25) and $P_{f}$, are selected mainly for the convenience of computer simulation. In practice, the covariance matrix usually possesses a more complex structure, while the probability of false alarm could be as low as $P_{f}=10^{-6}$. For each Monte-Carlo trial, the covariance matrix $\mathbf{R}$ is generated from an inverse Wishart distribution with mean $\overline{\mathbf{R}}$, and then, the covariance matrix $\mathbf{R}_{0}$ is generated by multiplying $\mathbf{R}$ with a scaling factor $\lambda$, i.e., $\mathbf{R}_{0}=\lambda \mathbf{R}$.

\section{A. Scale Invariance}

In the first example, we examine the invariance of various detectors with respect to the scaling factor $\lambda$. Among the examined detectors are Kelly's GLRT [1], the AMF [2], the standard ACE [5], the KA-GLRT [4], and the KA-AMF [4]. In this case, the threshold subject to the probability of false alarm $P_{f}=0.01$ is obtained from Monte Carlo simulations, when the scaling factor varies from $\lambda=1$ to $\lambda=16$ in a step size of 2 .

Fig. 1 shows the threshold of several detectors as a function of the scaling factor $\lambda$ when the number of training signals is $K=32$ and the SNR is $25 \mathrm{~dB}$. As illustrated, the standard ACE and the proposed KA-ACE have a constant threshold independent of $\lambda$ and, hence, is scale-invariant to the power scaling factor. By contrast, the thresholds of the AMF and the KA-AMF increase linearly as $\lambda$ increases, and the GLRT and the KA-GLRT give thresholds with two distinct phases: a gradually increasing phase when $\lambda$ is small; and a saturated phase when $\lambda$ is large, e.g., $\lambda>10$ in this example. 


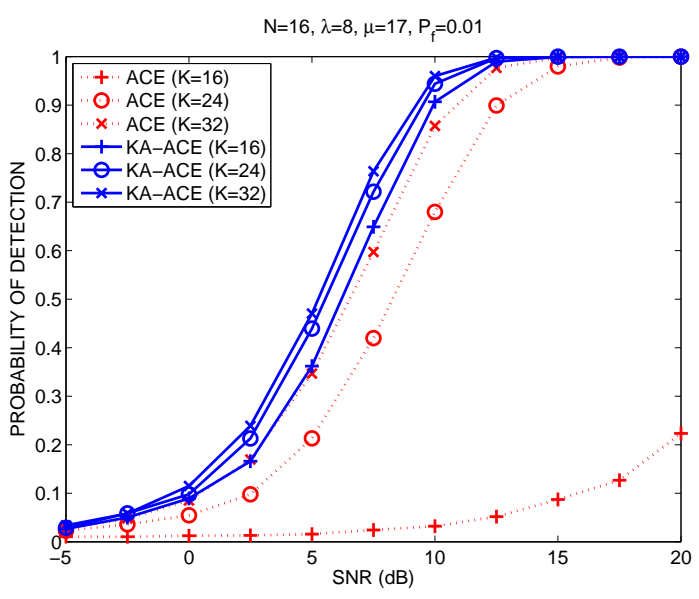

(a)

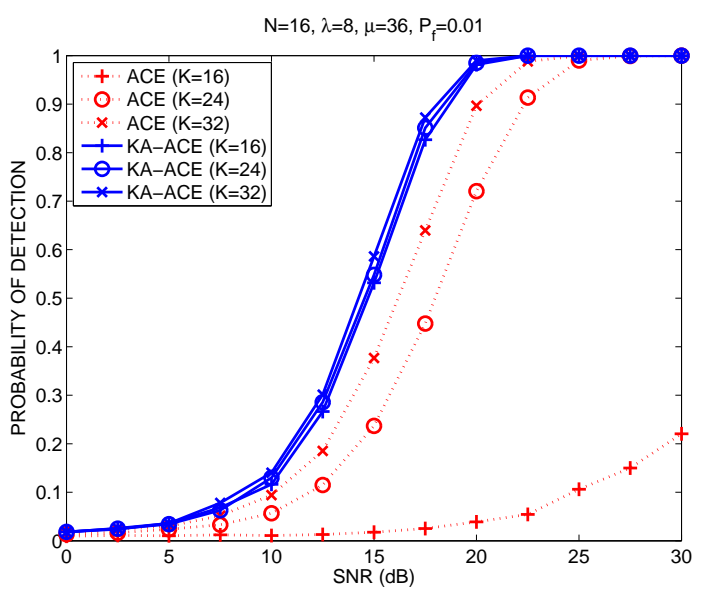

(b)

Fig. 2. Probability of detection versus SNR for different $K$ when $N=16, \lambda=8$, and $P_{f}=0.01$ for cases of (a) $\mu=17$; (b) $\mu=36$.

\section{B. Performance of Detection}

For the detection performance comparison, we consider the $\mathrm{ACE}$ and the KA-ACE detectors, which are scale-invariant to $\lambda$. Two cases, $\mu=17$ and $\mu=36$, are considered, which correspond to scenarios with less reliable and more accurate prior knowledge, respectively. In either case, we consider three training sizes, $K=16, K=24$, and $K=32$, are used. Note that $K=16$ is the minimum training size for the ACE to ensure the sample covariance matrix is full rank.

Fig. 2(a) shows the probability of detection versus SNR when the prior $\overline{\mathbf{R}}$ is less reliable, i.e., $\mu=17$. In this case, the knowledge-aided color loading in (19) put less weights on the prior matrix $\overline{\mathbf{R}}$. As seen from Fig. 2(a), in all cases, the KAACE has better detection performance than the standard ACE. Particularly, when $K=32$ and $P_{d}=0.8$, the performance gain of the KA-ACE over the ACE is about $1.5 \mathrm{~dB}$, while the marginal gain becomes more evident when the number of training signals is smaller, i.e., $K=24$ and $K=16$.

Fig. 2(b), on the other hand, shows the probability of detection versus SNR when the prior knowledge $\overline{\mathbf{R}}$ is more accurate, i.e., $\mu=36$. The results confirm that the KAACE has better performance than the ACE for the three training sizes considered. When $K=32$ and $P_{d}=0.8$, the performance gain of the KA-ACE over the ACE is about $2.1 \mathrm{~dB}$, larger than that of Fig. 2(a), which is because the prior knowledge is more accurate when $\mu=36$. Comparison between Figs. 2(a) and (b) also reveals that more training signals are more helpful to improve the detection performance when $\mu=17$, i.e., the prior knowledge is not so reliable.

\section{CONCLUSiON}

We introduced a stochastic model for adaptive detection in partially homogeneous environment. The model can incorporate a prior knowledge and handle clutter power variation between the test and training signals. The proposed KA$\mathrm{ACE}$ detector has the same form as the conventional ACE except that the former employs a linear combination of the sample covariance matrix and the prior covariance matrix for whitening, and the combining coefficients take into account the accuracy of the prior knowledge. Simulation results show that the KA-ACE offers better probability of detection than the $\mathrm{ACE}$ in cases of sufficient and, respectively, limited training signals. A future direction is to examine adaptive selection of parameter $\mu$, which indicates the significance of the prior covariance matrix, in the proposed stochastic model.

\section{REFERENCES}

[1] E. J. Kelly, "An adaptive detection algorithm," IEEE Transactions on Aerospace and Electronic Systems, vol. 22, no. 1, pp. 115-127, March 1986.

[2] F. C. Robey, D. R. Fuhrmann, E. J. Kelly, and R. Nitzberg, "A CFAR adaptive matched filter detector," IEEE Transactions on Aerospace and Electronic Systems, vol. 28, no. 1, pp. 208-216, January 1992.

[3] L. Svensson and M. Lundberg, "On posterior distributions for signals in Gaussian noise with unknown covariance matrix," IEEE Transactions on Signal Processing, vol. 53, no. 9, pp. 3554-3571, September 2005.

[4] A. De Maio, A. Farina, and G. Foglia, "Knowledge-aided Bayesian radar detectors \& their application to live data," IEEE Transactions on Aerospace and Electronic Systems, vol. 46, no. 1, pp. 170-183, February 2010.

[5] S. Kraut and L. L. Scharf, "The CFAR adaptive subspace detector is a scale-invariant GLRT," IEEE Transactions on Signal Processing, vol. 47, no. 9, pp. 2538-2541, September 1999.

[6] O. Besson, L. L. Scharf, and S. Kraut, "Adaptive detection of a signal known only to lie on a line in a known subspace, when primary and secondary data are partially homogeneous," IEEE Transaction on Signal Processing, vol. 54, no. 12, pp. 4698-4705, December 2006.

[7] M. Casillo, A. De Maio, S. Iommelli, and L. Landi, "A persymmetric GLRT for adaptive detection in partially-homogeneous environment," IEEE Signal Processing Letters, vol. 14, no. 12, pp. 1016-1019, December 2007.

[8] A. De Maio and S. Iommelli, "Coincidence of the Rao test, Wald test, and GLRT in partially homogeneous environment," IEEE Signal Processing Letters, vol. 15, no. 4, pp. 385-388, April 2008.

[9] O. Besson, J.-Y. Tourneret, and S. Bidon, "Knowledge-aided Bayesian detection in heterogeneous environment," IEEE Signal Processing Letters, vol. 14, no. 5, pp. 355-358, May 2007.

[10] S. Bidon, O. Besson, and J.-Y. Tourneret, "The adaptive coherence estimator is the generalized likelihood ratio test for a class of heterogeneous environments," IEEE Signal Processing Letters, vol. 15, no. 2, pp. 281-284, February 2008.

[11] S. Bidon, O. Besson, and J.-Y. Tourneret, "A Bayesian approach to adaptive detection in non-homogeneous environments," IEEE Transactions on Signal Processing, vol. 56, no. 1, pp. 205-217, January 2008.

[12] J. Ward, "Space-time adaptive processing for airborne radar," Technical Report 1015, Lincoln Laboratory, MIT, December 1994. 